\title{
SOLAR/INTERPLANETARY ENERGETIC PARTICLES IN THE OUTER HELIOSPHERE
}

\author{
Frank B. McDonald ${ }^{1}$ and Richard S. Selesnick ${ }^{2}$ \\ 1. Institute for Physical Science and Technology, U. Maryland, College Park, MD. \\ 2. California Institute of Technology, Pasadena, CA.
}

\begin{abstract}
The intense solar activity that occurred in the 1988/89 solar maximum period of cycle 22 produced long-lived solar/interplanetary energetic particle events that were observed out to 50 A.U. This paper presents a phenomenological view of these events and their relation to energetic particle increases in the previous cycle.
\end{abstract}

I. Introduction. There is a slow but dramatic evolution in the nature of solar energetic particle events with increasing heliocentric distance. At the Sun, solar flares frequently accelerate energetic ions to kinetic energies, $\mathrm{T}>10 \mathrm{MeV}$. In addition the flare may release a significant part of its energy in the form of a large-scale interplanetary shock wave. When observed at $1 \mathrm{AU}$, the energetic particles ejected from the flare-site display a rapid rise to peak intensity $(\sim 1 \mathrm{hr}, \mathrm{T}=10 \mathrm{MeV})$ followed generally by a relatively smooth exponential decay (several days). The flare shock traversing an event further accelerates low-energy particles and can modify the particle time-history at a given radial distance. These solar/interplanetary energetic particle events show a steady evolution with increasing heliocentric distance. Inside some 5AU, they retain the characteristics of individual solar particle events (Hamilton 1977, Zwickl and Webber 1977). By 10AU, the particle enhancements may extend over several solar rotations and display multiple shockassociated features and 26 day periodicities. The energy spectra reflects the presence of solar energetic particles at energies greater than some $30 \mathrm{MeV}$ as well as the shockaccelerated particles at lower energies. Beyond some $20 \mathrm{AU}$ most solar/IP particle increases show a relatively flat plateau structure at $\mathrm{T} \sim 4 \mathrm{MeV}$ that may persist over several solar rotations. In many cases these plateau regions are bounded by shock systems.

In the interplanetary medium there are interplanetary flow systems, the merged interaction regions (Burlaga et al. (1984) produced by the entrainment and coalescence of interplanetary transients, flare-produced shocks, high speed solar wind streams and coronal mass-ejections. The large-scale or global MIR's are closely related to the longterm modulation of galactic cosmic-rays and are generally associated with the long-lived energetic particle events in the outer heliosphere.

The origin of the $\mathrm{MeV}$ ions in these extended energetic particle increases in the outer heliosphere has not been established. They could be low-energy solar cosmic rays that are further accelerated by the MIR associated shocks; they could be similar to the co-rotating interaction region events where shocks from high speed solar wind streams accelerate suprathermal ions of the solar wind to $\mathrm{MeV}$ energies (McGuire et al. 1978, Gloeckler et al. 1979); or, they could represent a combination of these sources. These events are important in understanding the large scale structure of interplanetary disturbances in the outer heliosphere and they provide a means of studying particle acceleration by long-lived shock systems in an astrophysical plasma.

In this paper the Voyager and Pioneer energetic particle data are used to give an over-all phenomenological view of the energetic particle increases observed between 25 and 50AU associated with the intense 1989,90 solar activity. 
II. Observations. The level of solar activity from December 1988 to mid 1990 was very high ranking cycle 22 as the second most active in recorded solar history. At that time Voyager 2 and Pioneer 11 were near $30 \mathrm{AU}$ with $\mathrm{P}-11$ at a heliolatitude of $18^{\circ}, \mathrm{P}-10$ was near $45 \mathrm{AU}$ and $\mathrm{V}-1$ was essentially at the mid-point between them at a heliolatitude of $34^{\circ}$. As viewed in the distant heliosphere, the many solar energetic particle increases were coalesced into four events that extended from January 1989 well into the last half of 1990 (Fig. 1). On a broad scale, the time histories are quite similar at all locations but with several important exceptions. Event 1 is much smaller at V-1. The large increase that peaks around day 332 at $\mathrm{P}-10$ is produced by an exceptionally large shock that may be associated with a mid-August sea-level solar cosmic ray event. This shock is not observed by the other three spacecraft. The termination of the plateau region of event III at P-10 is marked by a second large shock that traces back to the great solar event of 29 September 1989. These very strong shocks are similar to that observed at P-10 in late July 1982 (Pyle, et al. 1984) and indicates the longitudinal asymmetries that a single strong solar event can produce. Event 2 is marked by the presence of protons of energies $>$ $60 \mathrm{MeV}$ and 2-8 MeV electrons at $30 \mathrm{AU}$ (Fig. 2). The comparative time histories and steep proton energy spectra suggest that the $\mathrm{MeV}$ electrons and $>30 \mathrm{MeV} \mathrm{H}$ received most of their energy at the flare site. However at lower energies, $(<5 \mathrm{MeV})$, anisotropies in the flow pattern indicate that acceleration processes are still occurring. A detailed study of these anisotropies using data from the V-2 low energy telescopes is now in progress. Preliminary analysis indicates there are periods when the anisotropies are surprisingly large.

The spectra for selected time intervals in events II and III (Fig. 3) are best fit with an exponential in rigidity similar to that characteristic of the CIR events (Van Hollebeke et al. 1978, Gloeckler et al. 1979) at smaller heliocentric distances. As Fisk and Lee (1979) have shown this spectral form can be produced by a combination of continuing shock acceleration and the adiabatic energy losses that occur in the expanding solar wind.

To examine the influence of particle intensity on heliolatitude and distance on particle intensity, 6 "equivalent" time intervals have been identified in events I-III for the 4 spacecraft and the 3.3-5 MeV H intensity has been plotted as a function of heliocentric distance (Fig. 4). The Pioneer 10 and 11 data are used to measure the radial gradient and the scatter of the V-1/2 data is taken as a measure of the intensity variation with heliolatitude. The radial gradients are on the order of $8-12 \%$. The small scatter of the V-2 data with respect to P-11 and the somewhat larger random scatter of the V-1 data suggest there are no large-scale changes with heliolatitude. What is surprising in this very simplistic approach is the apparent absence of any significant variations with heliolongitude (except for the transient asymmetries introduced by the passage of 2 exceptionally large interplanetary shocks, which has been excluded from the gradient studies). Within the range of observation these events represent extended shells propagating out into the distant heliosphere. However if these radial gradients are maintained at greater distances, the event intensities will approach the galactic flux levels (solar max levels) at $\sim 120$ A.U.

It is of interest to examine the long-term variation of solar/interplanetary energetic particles in the outer heliosphere. In Fig. 5 the P-10 and $113.3-5 \mathrm{MeV}$ intensities (26 day Avg.) are shown for the 1974-1989 period. The 1988-89 particle increases in the outer heliosphere are comparable to the peak intensities of the previous cycle (Fig. 5) (assuming a 10\%/A.U. gradient) although the 1888-89 $1 \mathrm{~A}$.U. events were larger and more frequent. It is probable that the integrated $\mathrm{P}-10$ intensities in the latter period were substantially larger in cycle 22 . The other very significant feature is the paucity of energetic particle increases in the 1976-77 and 1986-87 solar minimum intervals. Except 


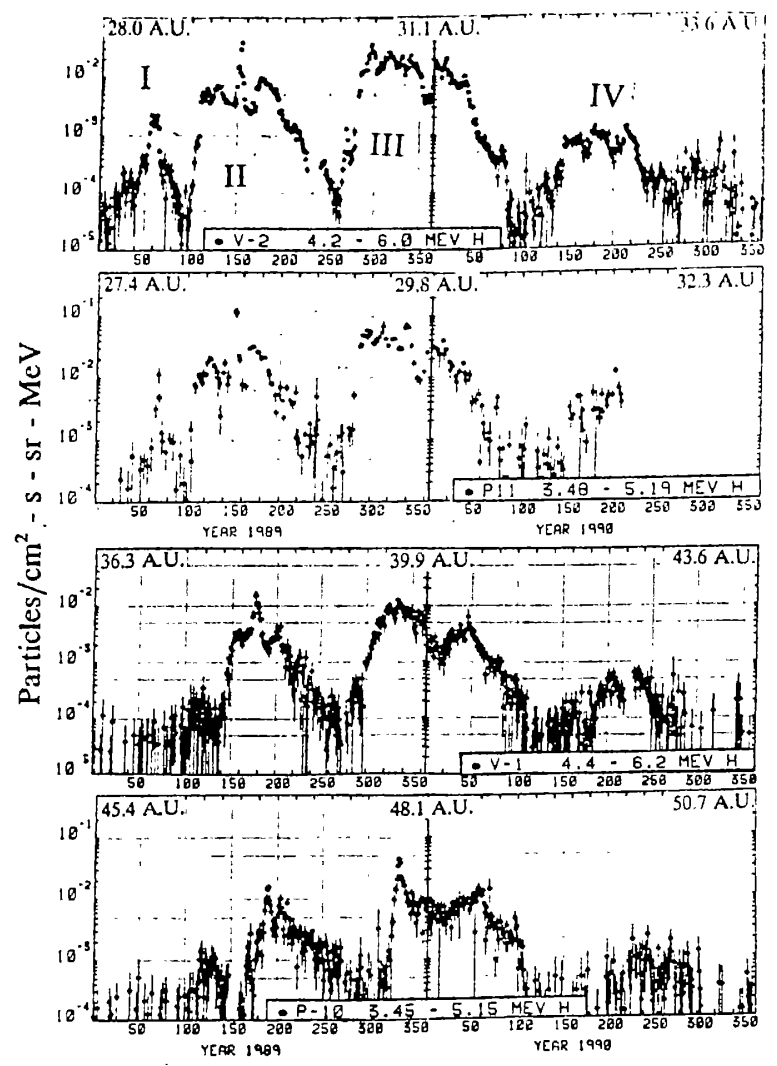

Fig. 3. $P-10 / 11$ rigidity spectra for 2 periods in events II and III (Fig. 1). The low energy increase in P-11 (II-2 and III-2) suggest shock acceleration in the outer heliosphere.

Fig. 1. 1989/90 Time histories for MeV Protons (24 hr $A V G$ ) for $P 10 / 11$ and $V 1 / 2$.
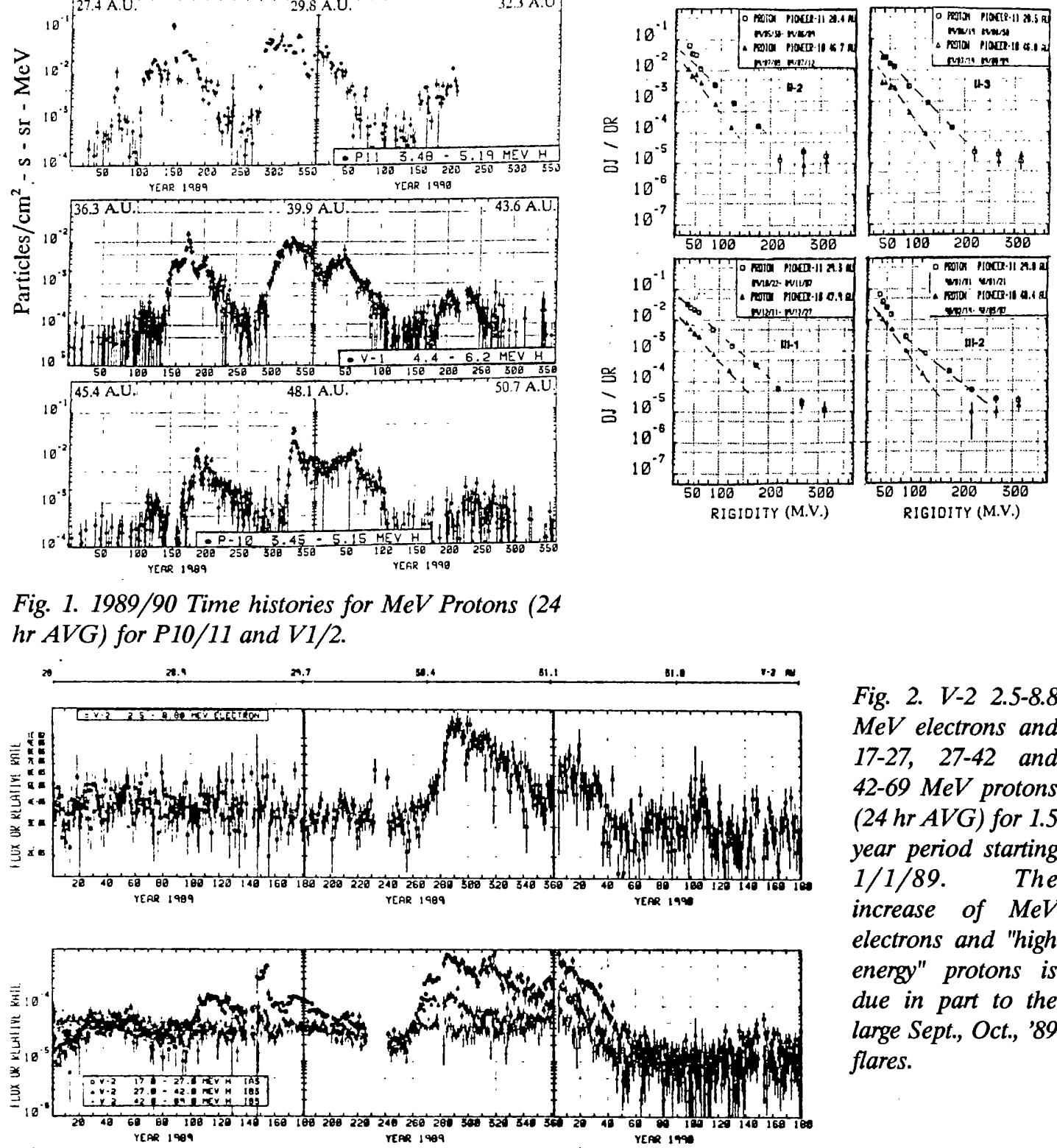

Fig. 2. V-2 2.5-8.8 $\mathrm{MeV}$ electrons and 17-27, 27-42 and 42-69 MeV protons (24 $\mathrm{hr} A V G)$ for 1.5 year period starting 1/1/89. The increase of $\mathrm{MeV}$ electrons and "high energy" protons is due in part to the large Sept., Oct., ' 89 flares.

for these periods, low energy solar/interplanetary energetic particles are almost continuously present in the outer heliosphere. These particles should be observed near 1 A.U. and are probably the source of the turn-up in the low-energy "quiet-time" spectra observed at 1 A.U. (Fan et al. 1966). 


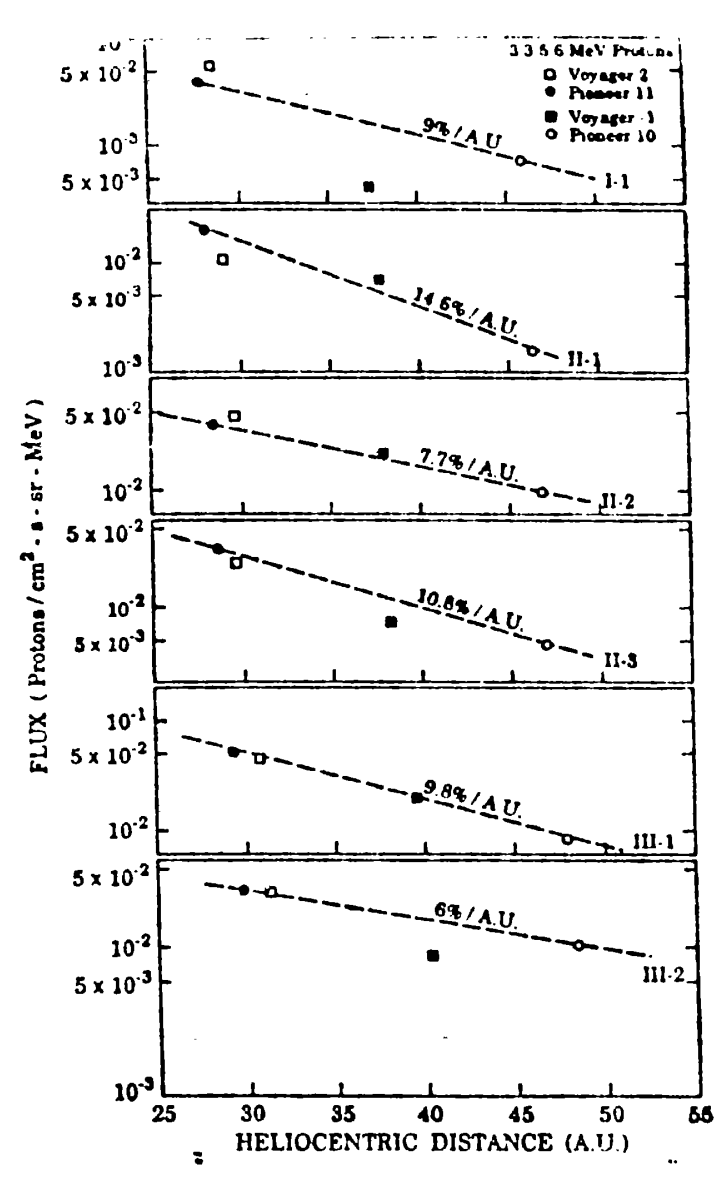

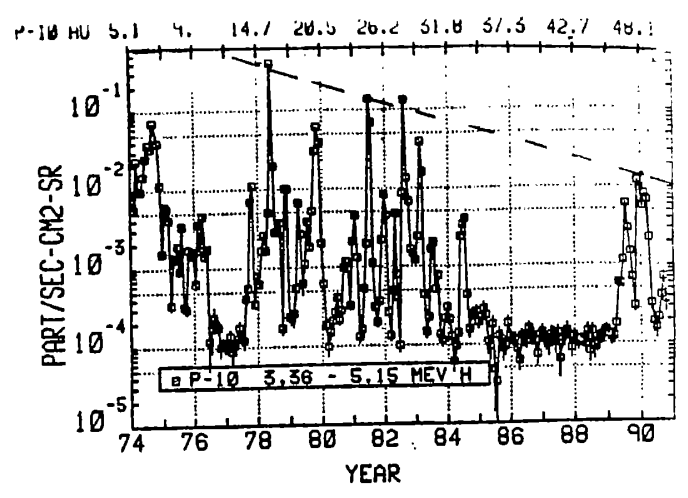

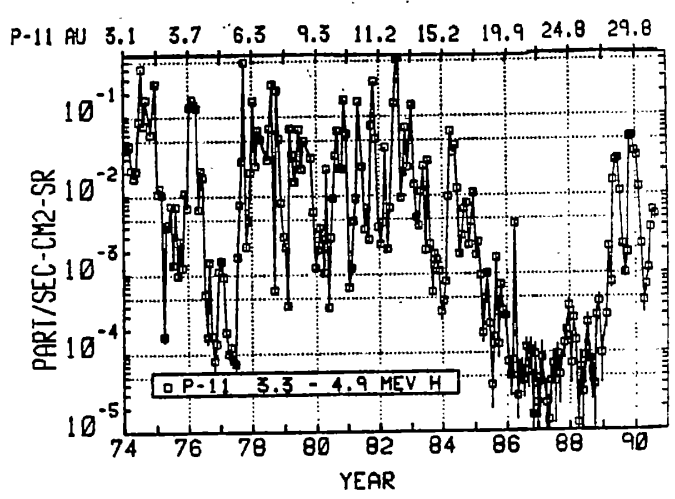

Fig. 5. Long-term (1974-1990.5) variations (26 day $A V G$ ) of 3.3-5 MeV protons in the outer heliosphere. The dashed line in the P-10 data is the intensity decrease expected for $a$ $10 \% / A . U$. radial gradient.

Fig. 4. Estimate of radial gradient using identical detectors $\quad . \quad$ P10 and $11\left(\lambda=17^{\circ}\right)$

$$
\text { . The lack }
$$

of systematic scatter in the Voyager $2(\lambda \approx 0)$ and $V$ $I\left(\lambda=34^{\circ}\right)$ suggest. $\cdot$ that the latitudinal gradients are small.

\section{References.}

Burlaga, L.F., McDonald, F.B., et al., 1984, JGR, 89, 6579.

Fan, C. ' G. Gloeckler, and J.A. Simpson 1966, Proc. 9th ICRC (London), 1, 109.

Gloeckler, G., D. Hovestadt, and L.A. Fisk 1979, Ap. J., 230, L191.

Hamilton, D.C. 1977, JGR, 82, 2157.

McGuire, R.E., T.T. Rosenvinge, and F.B. McDonald 1978, Ap J., 224, L87.

Pyle, K.R., J.A. Simpson, A. Barnes and J.D. Mikalov 1984, Ap J., 282, L107.

Van Hollebeke, Ma.I, F.B. McDonald, J.H. Trainor, et al. 1978, JGR, 83, 4723.

Zwickl, R.D. and W.R. Webber 1977, Solar Physics, 54, 457. 